\title{
Reactive 4D printing of mechanically programmable liquid crystal elastomer actuators
}

Morgan Barnes

Rice University

ACS Spring Meeting 2020 


\section{Shape Shifting Materials}


Applications:

- On-demand tactile buttons for smartphones

- Surface roughness modulation for stealth fighter jets

- Artificial heart valve bands that grows with the recipient

- Self-pumping microfluidic valves 


\section{Liquid Crystal Elastomers}

\section{Polydomain LCE}



Aligned LCE






\section{D Printing LCE}

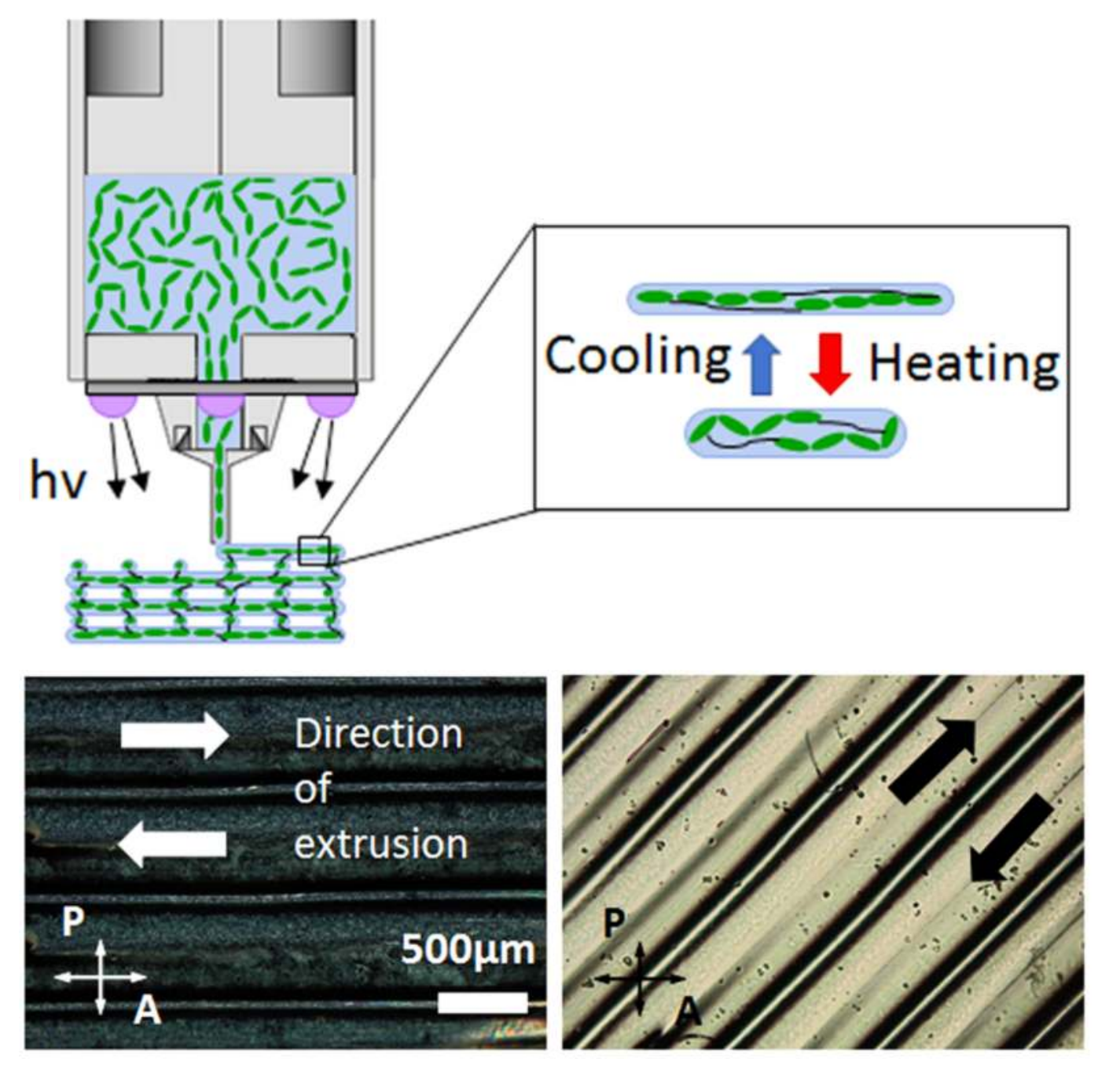

(a)



(d)



(g)



(b) $\mathrm{RT}$

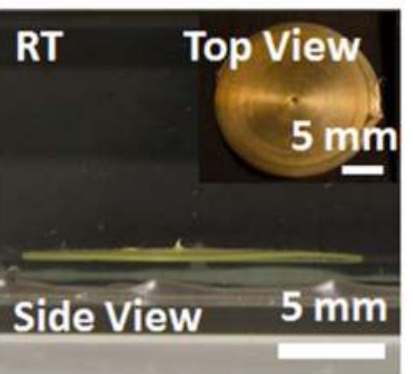

(e)

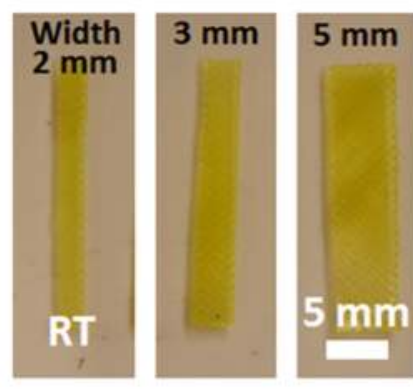

$160^{\circ} \mathrm{C}$



\section{Direct Shape Programming of LCEs}

Goal: Develop method to easily program complex and arbitrary shape changes into LCEs necessary for soft robotics or medical devices



First Network: Thiol-Acrylate Michael Addition Crosslinking



First Network

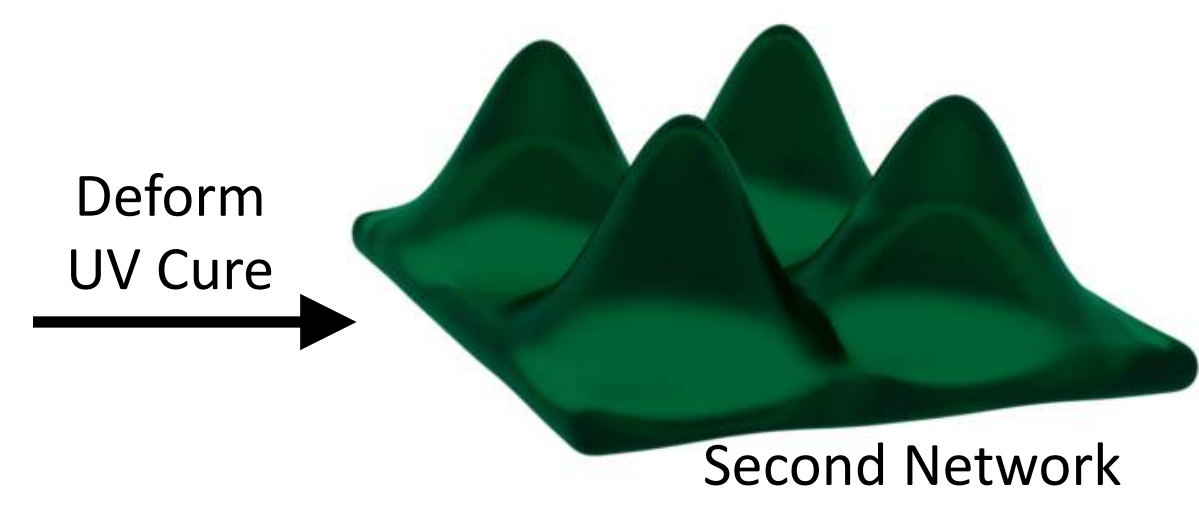

Second Network: Acrylate-Acrylate

Adapt two-stage thiol-acrylate LCE synthesis to 3D printing methods 


\section{Direct Shape Programming of LCEs}

Goal: Develop method to easily program complex and arbitrary shape changes into LCEs necessary for soft robotics or medical devices

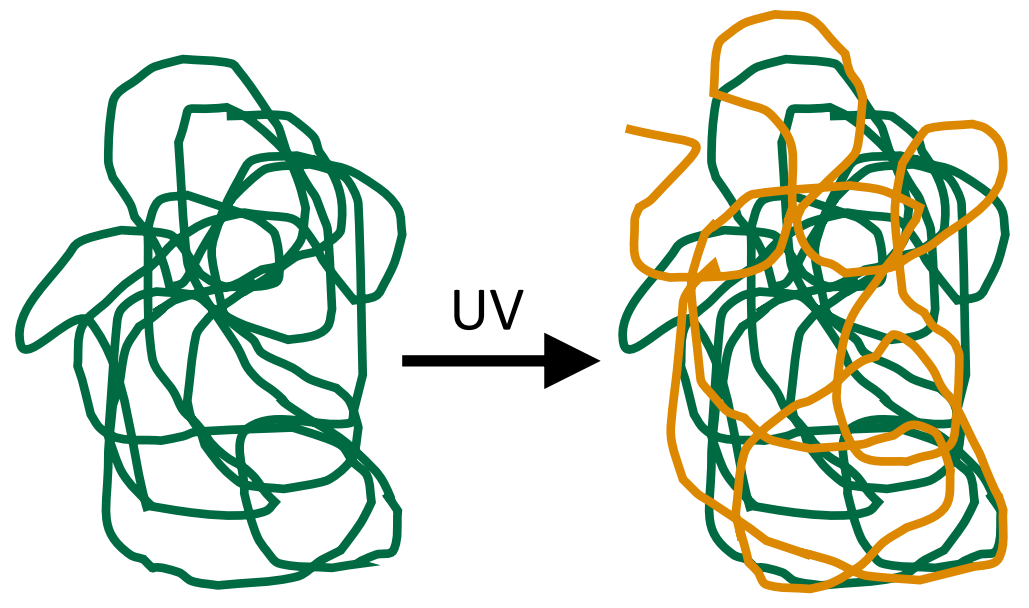

First Network: Thiol-Acrylate Michael Addition Crosslinking



Second Network: Acrylate-Acrylate

Adapt two-stage thiol-acrylate LCE synthesis to 3D printing methods 


\section{Reactive 3D Printing of LCES}

1. An LCE oligomer solution is prepared by partially completing a thiol-acrylate Michael addition

2. The solution is 3D printed in the desired shape in a catalyst bath to complete the Michael addition

3. Once the solvent is evaporated, the LCE is mechanically deformed and UV cured to complete the second network

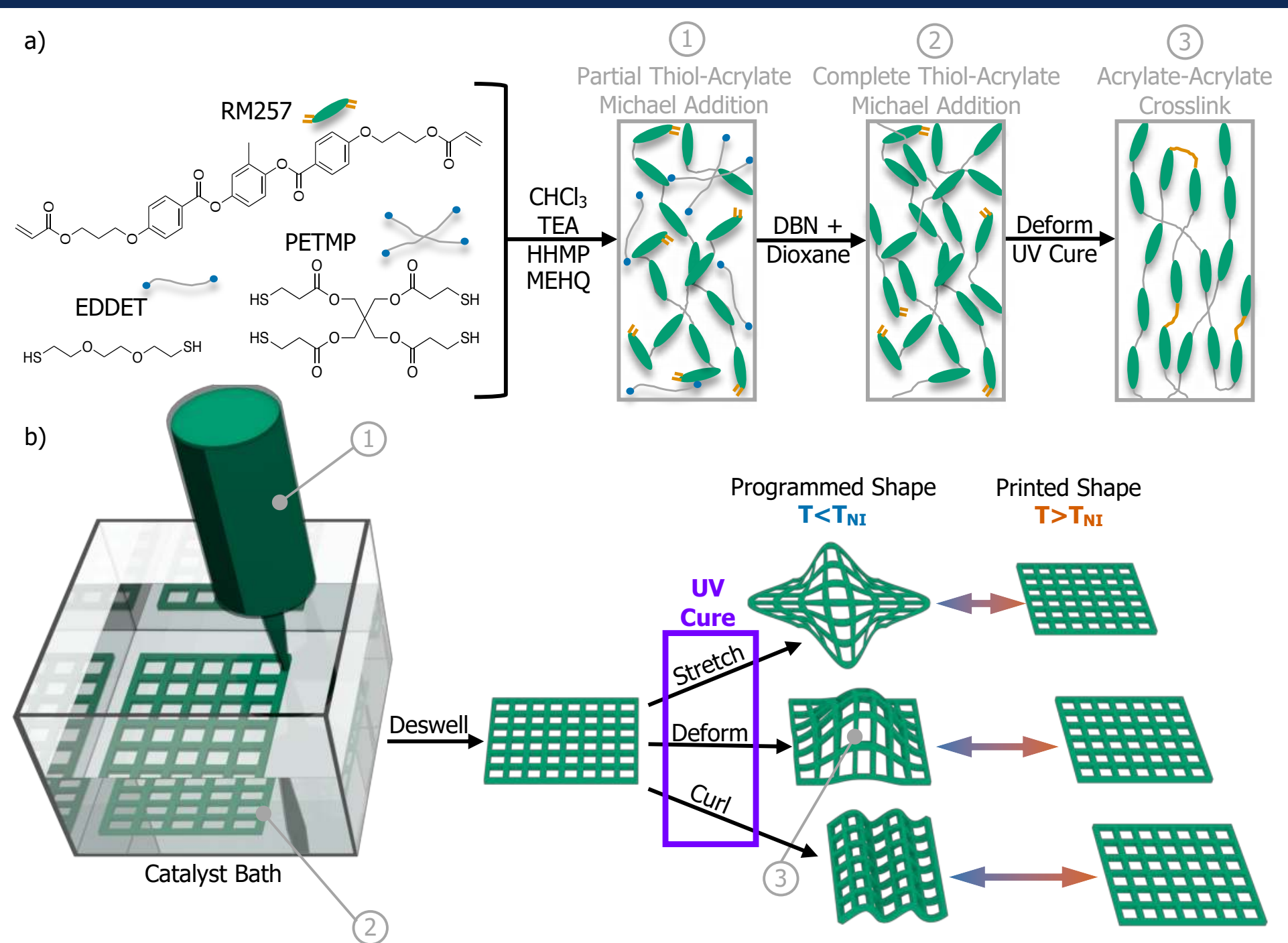




\section{D Printing Parameters}

\section{Oligomer Solution}



Catalyst Bath

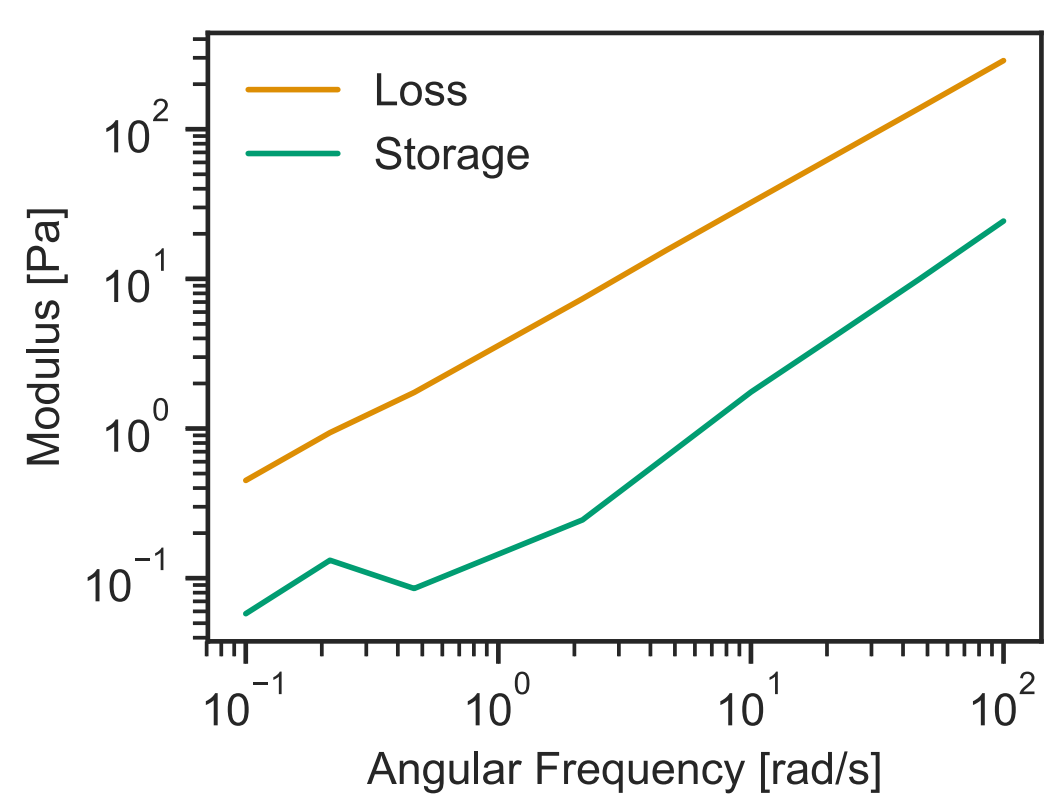

Solvents

Swelling

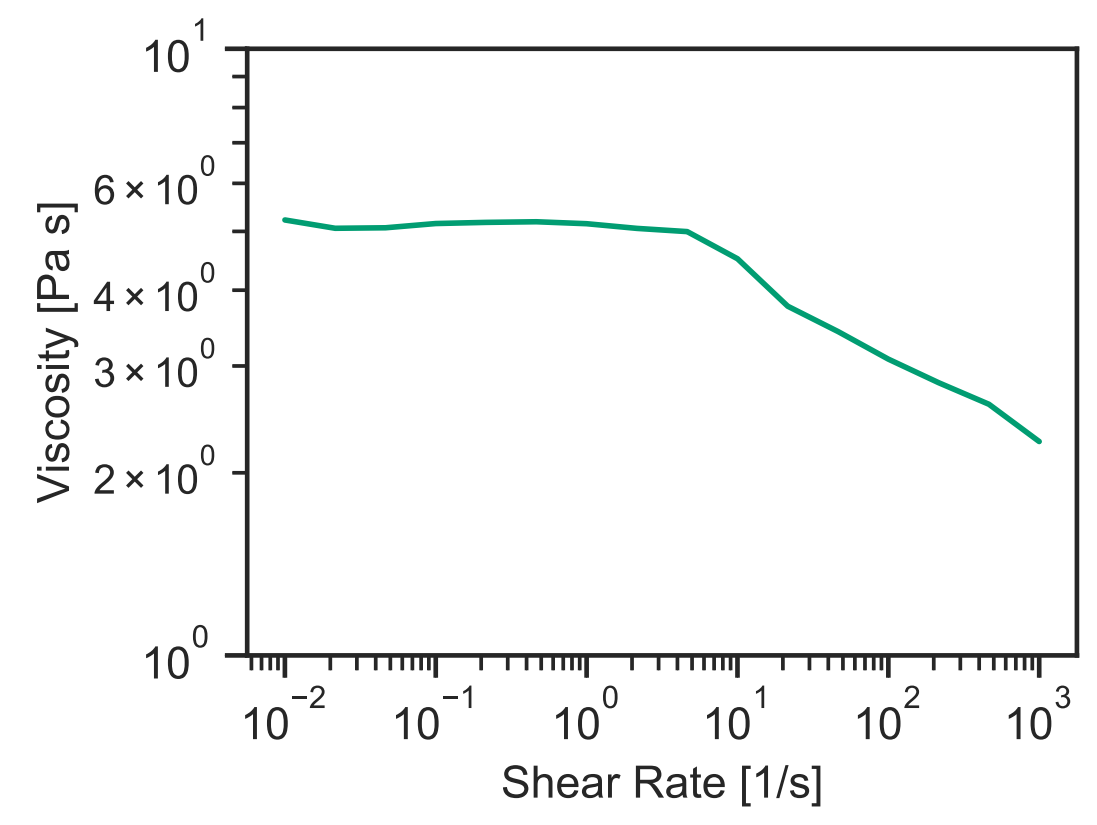

Oligomer solution is polymerized just below the critical gel point to enable rapid solidification upon printing into the catalyst bath. 


\section{D Printing Parameters}

Oligomer Solution<smiles>CCCCCCN</smiles>

HexAM<smiles>CCCNCCC</smiles>

DnPA
Catalyst Bath<smiles>CCN(CC)CC</smiles>

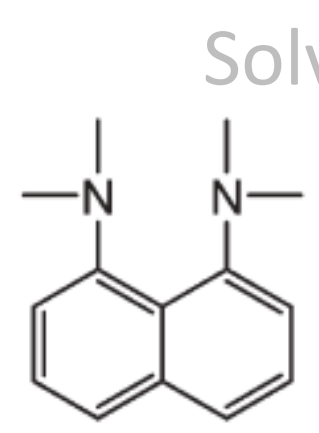

PS
Solvents<smiles>C1CCC2=NCCCN2CC1</smiles>

DBU
Swelling<smiles>C1CC2=NCCN2C1</smiles>

DBN catalyst

HexAM

DnPA

TEA

PS

DBU

DBN $\mathrm{k}_{\mathrm{app}} / 10^{-4}\left(\mathrm{~mol} \mathrm{~L}^{-1} \mathrm{~s}^{-1}\right)^{\mathrm{c}}$

53.4

8.02

0.028

0.02

$$
5.24
$$

54.9

- Only focused on tertiary amines to reduce unwanted side reactions

- TEA reaction too slow to print multi-layer structures

- DBN reaction is very quick (ideal) needs to be diluted with solvent to prevent instantaneous clogging of the extrusion nozzle during printing 


\section{D Printing Parameters}

Oligomer Solution



Printed Film
Catalyst Bath

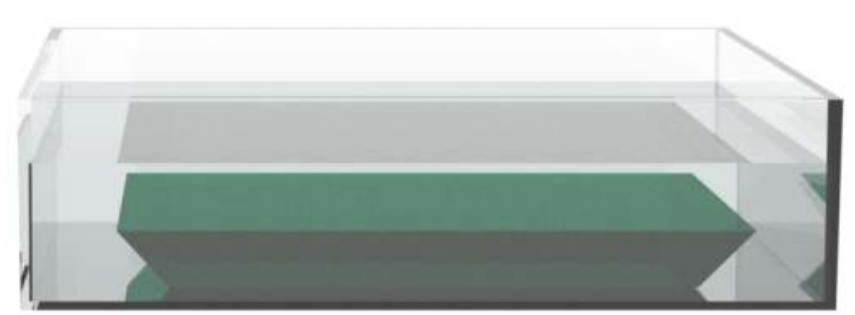

Anisotropic swelling from surface anchoring
Swelling



Anisotropic deswelling



Solution: Swell oligomer solution to just below the swelling amount of the LCE in the catalyst bath to prevent excessive swelling during printing. 


\section{Comparison of 3D Printed to Bulk LCE Films}

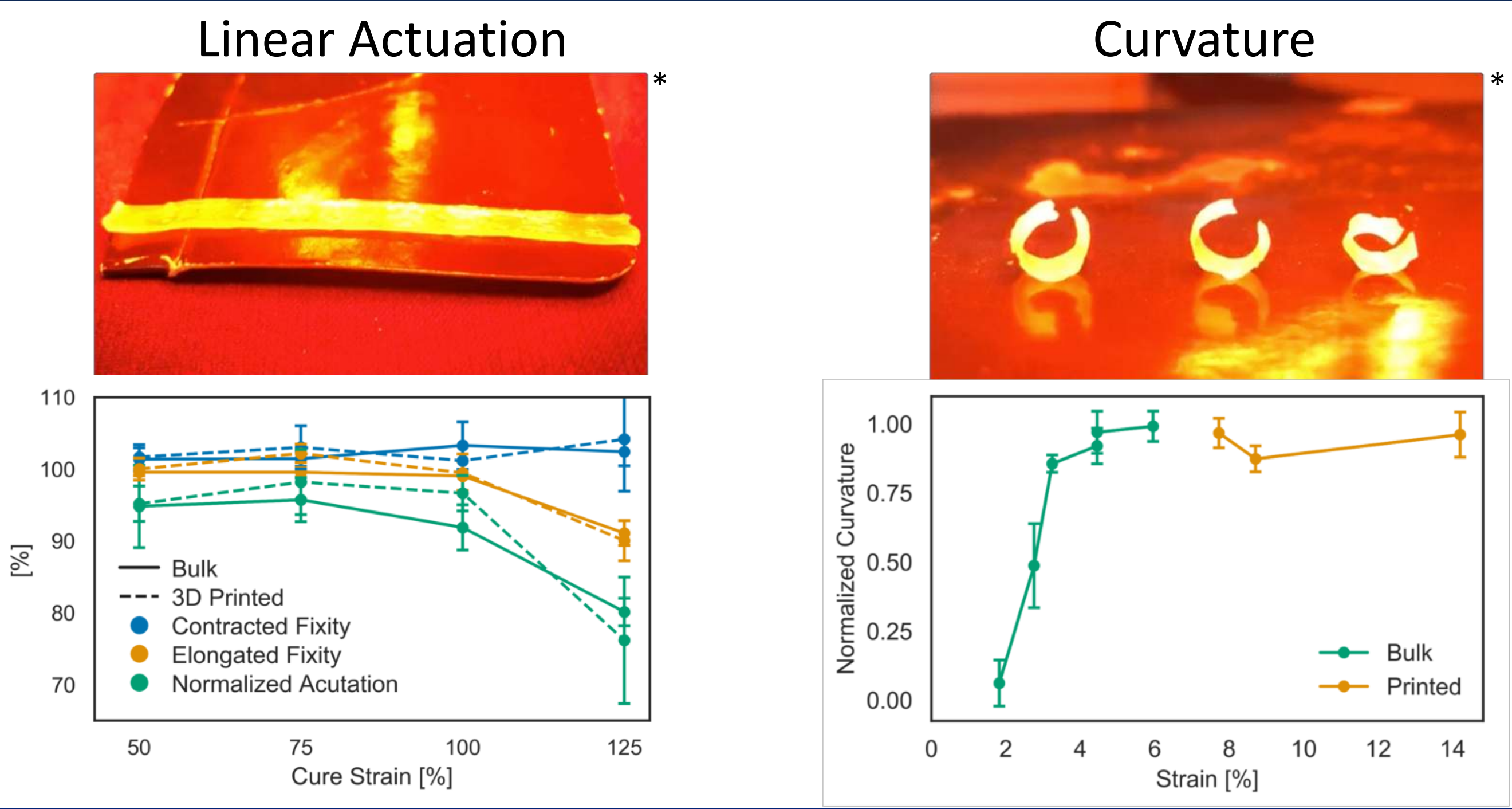




\section{Comparison of 3D Printed to Bulk LCE Films}

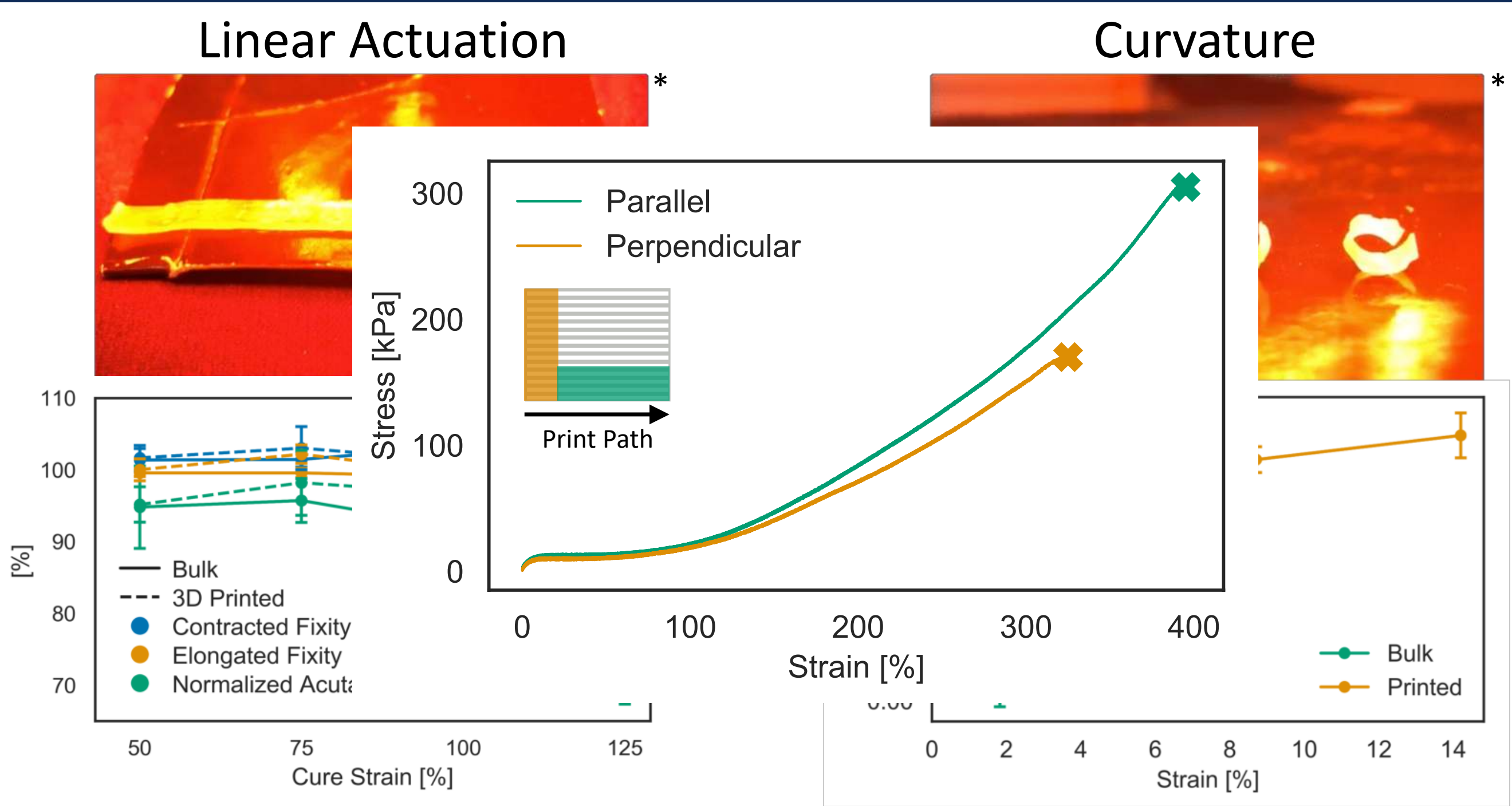


Arbitrary Shape Programming from a Single Printed Structure

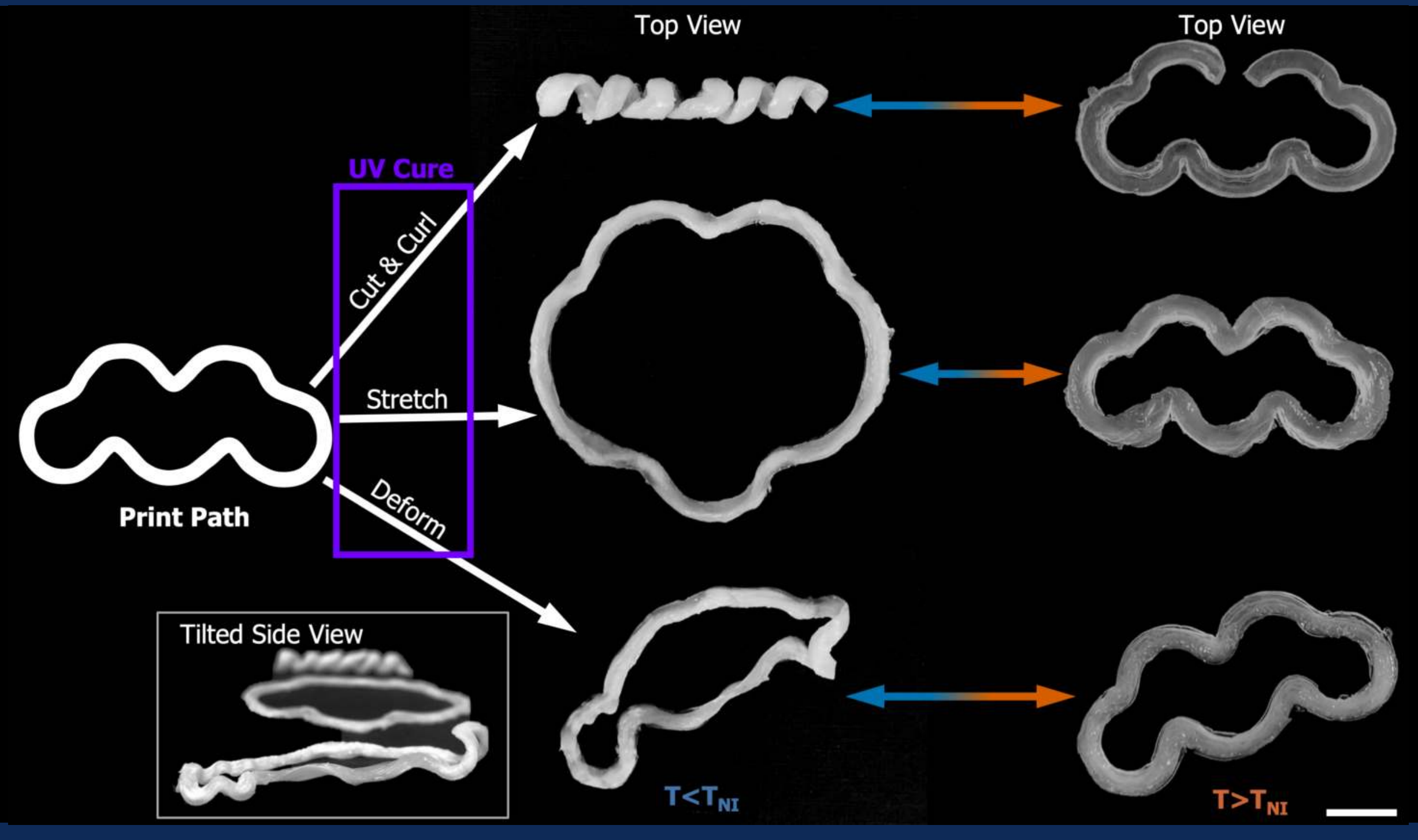




\section{Actuation of 3D Printed LCEs}
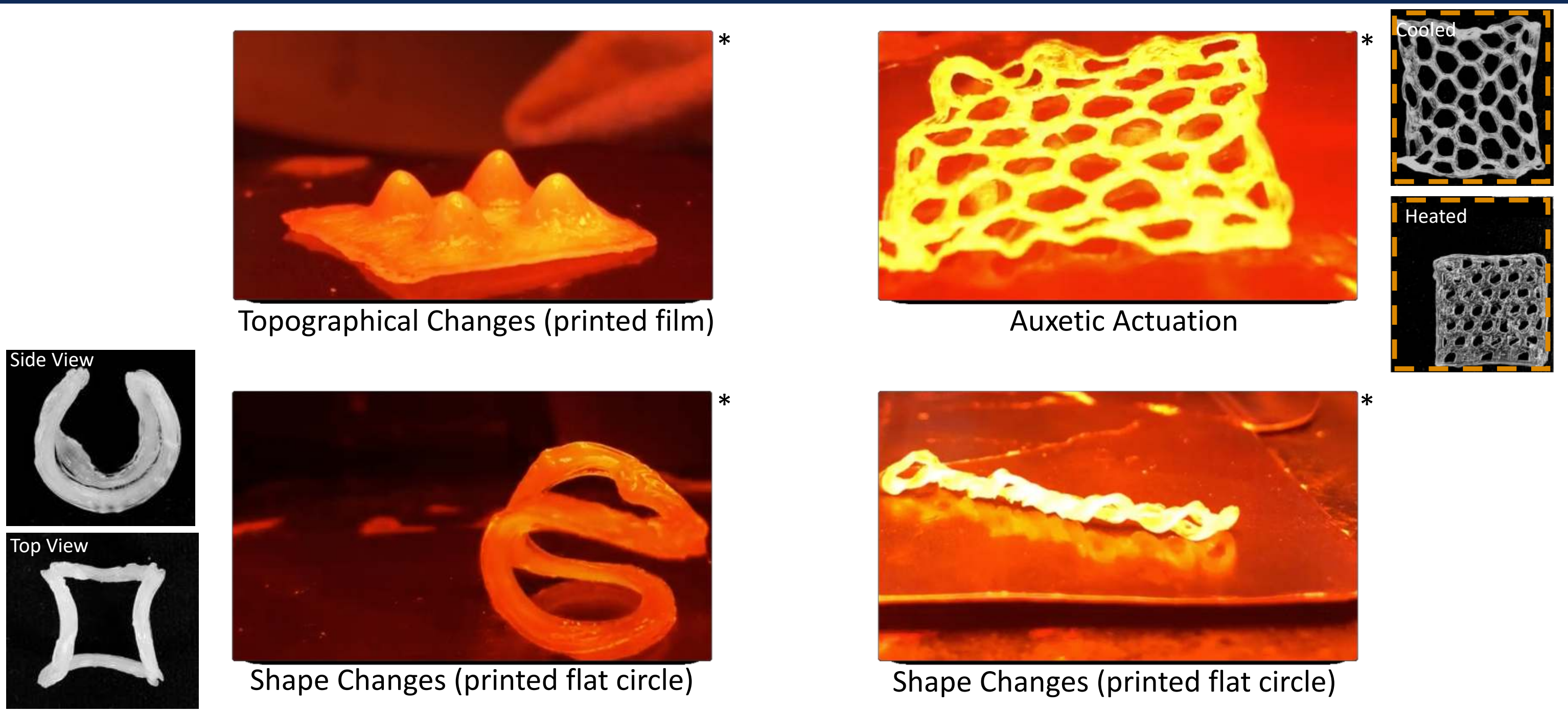

Shape Changes (printed flat circle)

Shape Changes (printed flat circle) 


\section{Conclusions and Future Work}

- Developed reactive 3D printing method that enables sequential base- and photo-catalyzed polymerizations

- Printed LCEs can be mechanically deformed and UV cured after printing to program desired shape change

- Does not rely on knowing the mathematical director profile needed for a shape change and decouples the printing and programming process

- Future work includes using scaffolds and printing optimization to develop complex 3D printed structures and to investigate dip molding fabrication methods 


\section{Acknowledgments}

\section{Verduzco Lab}

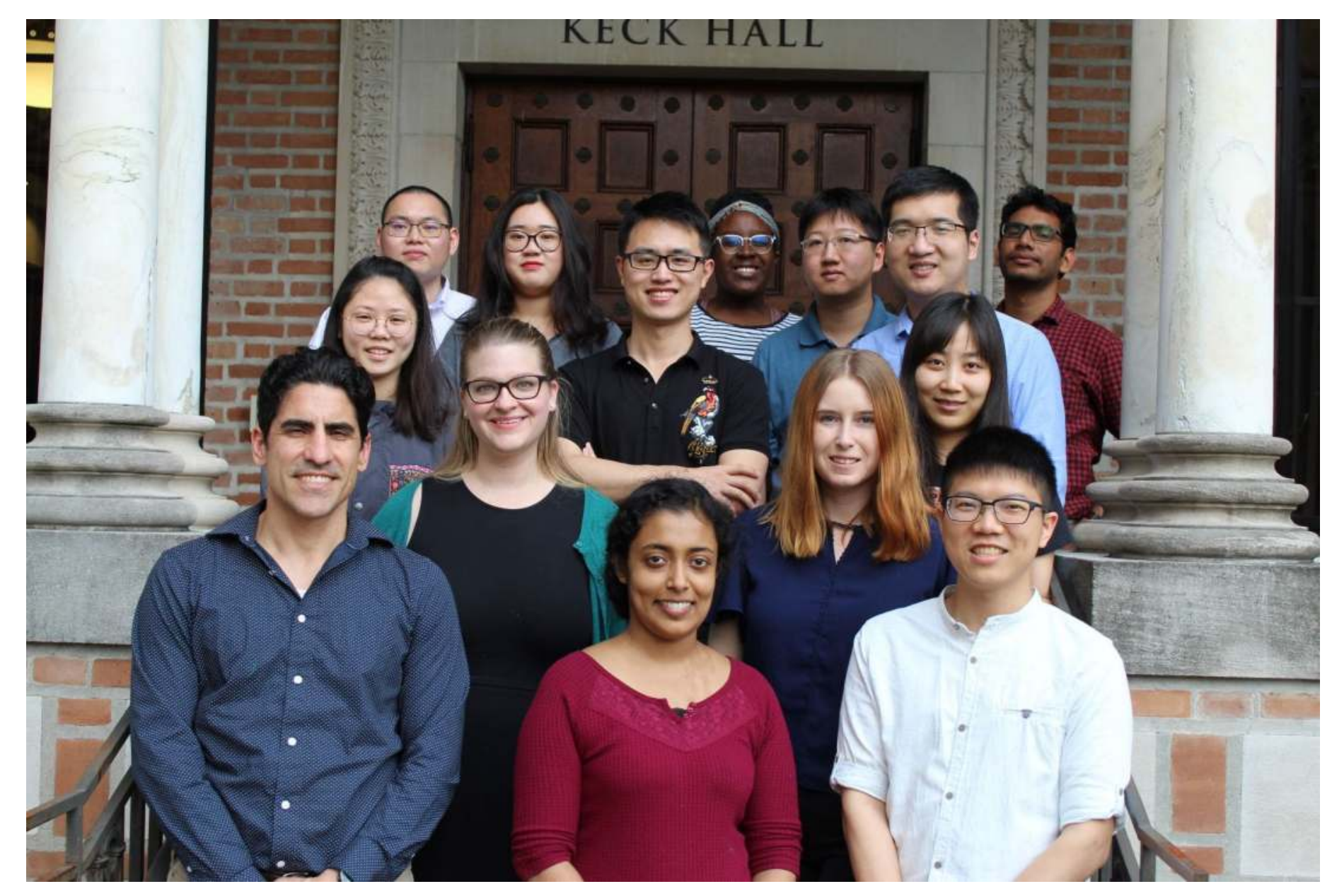

Shaan Parekh Maksud Rahman Mohammad Sayed

Pulickel Ajayan


@MGBarnes

MGBarnes.com

$\triangle$ Morgan.Barnes@rice.edu 